\title{
Case Report \\ Oxford-AstraZeneca Coronavirus Disease-2019 Vaccine-Induced Immune Thrombocytopenia on Day Two
}

\author{
Azhar Kareem Razzaq $\mathbb{D i}^{1}$ and Ameer Al-Jasim $\mathbb{D}^{2}$ \\ ${ }^{1}$ College of Medicine-University of Basrah, Department of Haematology, Baghdad, Iraq \\ ${ }^{2}$ College of Medicine-University of Baghdad, Baghdad, Iraq \\ Correspondence should be addressed to Azhar Kareem Razzaq; dr.azharalasady@gmail.com
}

Received 17 June 2021; Accepted 14 July 2021; Published 21 July 2021

Academic Editor: Pier Paolo Piccaluga

Copyright (C) 2021 Azhar Kareem Razzaq and Ameer Al-Jasim. This is an open access article distributed under the Creative Commons Attribution License, which permits unrestricted use, distribution, and reproduction in any medium, provided the original work is properly cited.

\begin{abstract}
Introduction. Vaccines have been one of the most impactful human discoveries that have significantly changed life expectancy. Immune thrombocytopenic purpura (ITP) is an autoimmune disease characterized by platelet damage, life-threatening thrombocytopenia, and haemorrhage when the platelet count reaches below $20 \times 10^{9} / \mathrm{mcL}$. Its pathogenesis involves viral mimicry or T-cell-induced immune destruction in antibody-negative cases. The clinical manifestations of thrombocytopenia vary according to the severity (level of platelets) and range from being asymptomatic to severe haemorrhage. ITP is treated with immunosuppression. Case Presentation. A 26-year-old Iraqi male laboratory analyst with an unremarkable medical history presented with severe thrombocytopenia 2 days after receiving the Oxford-AstraZeneca coronavirus disease-2019 vaccine. The patient was asymptomatic with unremarkable examination findings. However, his low platelet count was discovered accidentally, and the patient did not exhibit the resistance pattern of ITP and recovered successfully with regular immunosuppressant treatment. Conclusion. Patients with a history of thrombocytopenia can develop vaccine-induced thrombocytopenia earlier than the expected onset. Close monitoring, through regular complete blood counts, is highly recommended for patients with previous thrombocytopenia because the immune modulation process of the vaccine can worsen preexisting thrombocytopenia.
\end{abstract}

\section{Introduction}

Vaccines have become an impactful human discovery that has significantly changed life expectancy and disease incidence. However, adverse events and autoimmune phenomena, including immune thrombocytopenic purpura (ITP), have been reported in vaccination [1].

ITP is an autoimmune disease characterized by platelet damage that causes life-threatening thrombocytopenia through haemorrhage, when the platelet count drops below $20 \times 10^{9} / \mathrm{mcL}$. The pathogenesis of vaccine-associated autoimmunity most likely involves virally induced molecular mimicry or a T-cell immune-mediated mechanism $[2,3]$.

The clinical manifestations of ITP range from asymptomatic to light purpuric rash all over the body to severe intracranial haemorrhage [4]. The usual treatments for such conditions are immunosuppressants, such as steroids and intravenous immunoglobulin (IVIG) [2].

Different vaccines have reportedly caused ITP through various mechanisms, eventually leading to thrombocytopenia. Thus, it is not unusual for a vaccine to cause ITP [1]. On the 8th of June 2021, Welsh et al. reported the occurrence of immune thrombocytopenia with the Pfizer and Moderna vaccines in their manuscript regarding the COVID-19 Vaccine Adverse Event Reporting System. We report the first case of immune thrombocytopenia induced by the Oxford-AstraZeneca coronavirus disease-2019 (COVID-19) vaccine [5].

In this case, we present an Oxford-AstraZeneca COVID19 vaccine-induced immune thrombocytopenia that occurred on the second day in a 26-year-old man with a medical history of undiagnosed thrombocytopenia. 


\section{Case Presentation}

A 26-year-old Iraqi male laboratory analyst presented with severe thrombocytopenia two days after receiving the Oxford-AstraZeneca COVID-19 vaccine. He had an unremarkable medical history, except for mild thrombocytopenia, ranging from 100 to $130 \times 10^{9}$. He had not sought consult for a provisional diagnosis. In addition to experiencing mild headaches, the patient had generally been asymptomatic. His low platelet counts were discovered incidentally through a routine complete blood count.

On examination, the patient was well, conscious, oriented, not in distress, and vitally stable. There was no gum or conjunctival bleeding, epistaxis, petechiae, purpura, or ecchymosis. Jaundice, organomegaly, and lymphadenopathy were also not noted.

Further laboratory testing revealed a platelet count of $64 \times 10^{9} / \mathrm{L}$ on day 2 , which dropped to $35 \times 10^{9} / \mathrm{L}$ on day 3 . The rest of his complete blood count results were unremarkable. His fibrinogen level was $145 \mathrm{mg} / \mathrm{mL}$, and his prothrombin time, partial thromboplastin time, D-dimer, brain magnetic resonance imaging, and magnetic resonance venography were all normal.

The management plan consisted of hospital admission, methylprednisolone (pulse dose at $1 \mathrm{~g} / \mathrm{kg}$ for 3 days), and IVIG ( $1 \mathrm{~g} / \mathrm{kg}$ for 2 days).

The patient responded well to treatment and had a favourable outcome. On the fifth day, he had a platelet count of $90 \times 10^{9} \mathrm{~L}$ with a normal coagulation profile. His platelet count normalized in the second week. The patient was then discharged based on his improved blood count and was recommended for periodic follow-up.

\section{Discussion}

There have been at least 25 reports of ITP related to the COVID-19 vaccine. Reportedly, only the Pfizer and Moderna vaccines caused this adverse event. However, this side effect is not exclusively attributable to these vaccines [5-8].

Thrombocytopenia is not an unusual outcome of an immune modulation process, such as vaccination. It is expected to develop 4-30 days after vaccine administration [9]. In the present case, severe asymptomatic thrombocytopenia was noted 2 days after the Oxford-AstraZeneca COVID-19 vaccine had been administered. This contradicted the known onset and symptomatology of vaccine-induced thrombocytopenia. This deviation was attributed to the patient's undiagnosed thrombocytopenia.

The patient's good clinical response to the standard ITP regimen confirmed the immune-induced process of thrombocytopenia. This was most likely associated with the corresponding vaccine. The clinical scenario was similar to that of patients who received the Moderna and Pfizer vaccines $[5,8]$.

The deleterious effects of aggressive immunosuppression on vaccine-induced thrombocytopenia should not be overlooked. In prior studies, postvaccination immunosuppressive therapy attenuated the immune response to the administered vaccine [10].

\section{Conclusion}

In conclusion, patients with a history of thrombocytopenia can develop vaccine-induced thrombocytopenia earlier than the expected onset. Further studies are warranted to investigate this phenomenon.

Close monitoring of patients with previous thrombocytopenia is highly recommended because the immune modulation process of the vaccine can worsen the preexisting thrombocytopenia. Thus, regular complete blood count is indicated after COVID-19 vaccination.

\section{Data Availability}

The data used to support the findings of this study are available as gray literature (offline) and are available from the corresponding author upon request.

\section{Consent}

Written informed consent was obtained from the patient to publish his details.

\section{Conflicts of Interest}

The authors declare that there are no conflicts of interest.

\section{References}

[1] C. Perricone, F. Ceccarelli, G. Nesher et al., "Immune thrombocytopenic purpura (ITP) associated with vaccinations: a review of reported cases," Immunologic Research, vol. 60, no. 2-3, pp. 226-235, 2014.

[2] D. B. Cines and V. S. Blanchette, "Immune thrombocytopenic purpura," New England Journal of Medicine, vol. 346, no. 13, pp. 995-1008, 2002.

[3] M. Rinaldi, C. Perricone, O.-D. Ortega-Hernandez, R. Perricone, and Y. Shoenfeld, "Immune thrombocytopaenic purpura: an autoimmune cross-link between infections and vaccines," Lupus, vol. 23, no. 6, pp. 554-567, 2014.

[4] V. Saudagar, S. Patil, S. Goh, and S. Pothiawala, "Vigilance regarding immune thrombocytopenic purpura after COVID19 vaccine," Irish Journal of Medical Science, vol. 1971, pp. 1-2, 2021.

[5] K. J. Welsh, J. Baumblatt, W. Chege, R. Goud, and N. Nair, "Thrombocytopenia including immune thrombocytopenia after receipt of mRNA COVID-19 vaccines reported to the vaccine adverse event reporting system (VAERS)," Vaccine, vol. 39, no. 25, pp. 3329-3332, 2021.

[6] J. M. Helms, K. T. Ansteatt, J. C. Roberts et al., "Severe, refractory immune thrombocytopenia occurring after SARSCoV-2 vaccine," Journal of Blood Medicine, vol. 12, pp. 221224, 2021.

[7] E. J. Lee, D. B. Cines, T. Gernsheimer et al., "Thrombocytopenia following Pfizer and Moderna SARS-CoV-2 vaccination," American Journal of Hematology, vol. 96, no. 5, pp. 534-537, 2021.

[8] T. Shimabukuro, COVID-19 Vaccine Safety Update, Advisory Committee on Immunization Practices (ACIP), Atlanta, GA, USA, 2021.

[9] J. B. Bussel, J. M. Connors, and D. B. Cines, "Thrombosis with thrombocytopenia syndrome (also termed vaccine-induced 
thrombotic thrombocytopenia)," American Society of Hematology, vol. 25, 2021.

[10] K. A. Papp, B. Haraoui, D. Kumar et al., "Vaccination guidelines for patients with immune-mediated disorders on immunosuppressive therapies," Journal of Cutaneous Medicine and Surgery, vol. 23, no. 1, pp. 50-74, 2019. 